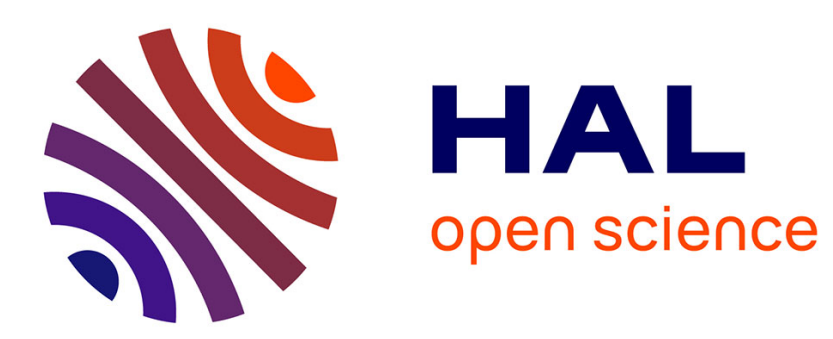

\title{
Quand "facilement" rencontre un numéral, ça parle d'approximation
}

Céline Vaguer

\section{To cite this version:}

Céline Vaguer. Quand "facilement" rencontre un numéral, ça parle d'approximation. L'information grammaticale, 2010, 125, pp.29-35. hal-00980131

\section{HAL Id: hal-00980131 \\ https://hal.science/hal-00980131}

Submitted on 17 Apr 2014

HAL is a multi-disciplinary open access archive for the deposit and dissemination of scientific research documents, whether they are published or not. The documents may come from teaching and research institutions in France or abroad, or from public or private research centers.
L'archive ouverte pluridisciplinaire HAL, est destinée au dépôt et à la diffusion de documents scientifiques de niveau recherche, publiés ou non, émanant des établissements d'enseignement et de recherche français ou étrangers, des laboratoires publics ou privés. 


\section{QUAND FACILEMENT RENCONTRE UN NUMÉRAL, ÇA PARLE D'APPROXIMATION}

\section{L'ART DE PARLER FLOU}

Il existe en langue des marqueurs qui permettent aux locuteurs de s'exprimer dans le vague, de "parler flou", soit qu'ils n'aient pas le moyen ou l'intention de fixer plus avant ce qu'ils veulent exprimer (Il habite aux environs de Troyes), soit que la langue elle-même soit moins précise que ce que peut laisser croire une première intuition : le départ dans On partira à $5 h$ n'est qu'en apparence exactement déterminé, comme le montrent On partira à $5 \mathrm{~h}$ précises ${ }^{1} /$ pétantes, On partira sur le coup de $5 \mathrm{~h}$. Ces marqueurs d'approximation ne sont pas nécessairement à interpréter comme l'indice d'un manque car ils jouent aussi un rôle dans le processus de communication, assurant par exemple une fonction d'atténuation (Caffi 1999).

Un tour d'horizon des travaux anglophones ${ }^{2}$ permet de mettre en évidence l'existence de deux grandes familles de marqueurs :

- les marqueurs d'approximation [hedges ${ }^{3}$ (Lakoff), approximators (Prince et al.), busches (Caffi)], qui prennent place dans le contenu logique de l'énonciation, et qui affectent de nuances (approximation, précision...) les termes avec lesquels ils se construisent. En rendant le contenu flou et moins précis, ces marqueurs réduisent indirectement l'engagement du locuteur vis-à-vis de son propos.

Parmi les marqueurs d'approximation, on distingue :

- d'une part ceux qui indiquent une lecture approximative d'une expression quantitative (les rounders de Prince et al.) : à peu près, environ, presque, près de, autour de, approximativement...

- d'autre part ceux qui indiquent une divergence entre le sens lexical de l'expression et son emploi; on peut parler d'approximation lexicale (les adaptors de Prince et al.) : quelque chose comme ça, plutôt, plus exactement, plus ou moins, (il est quelque part) par ici ${ }^{4}$, çà et là, sorte de, genre de, espèce de... qui affectent la valeur de vérité de la proposition, comme l'illustre (1) :

\section{(1) a. Le canari est un oiseau \\ b. Le canari est une sorte d'oiseau}

- les marqueurs d'attitude énonciative - que l'on pourrait qualifier de dérobade [hedges performatives (Fraser), shields (Prince et al.), hedges (Caffi)] qui se focalisent davantage sur la relation du locuteur avec son énonciation, et qui ont pour effet de réduire son engagement, de marquer une prise de distance de sa part, quant à la véracité de son propos.

\footnotetext{
${ }^{1}$ L'exemple On partira à 5 h précises ne dénote pas la précision mais la restriction. Ce qui tend à dire que l'énoncé $O n$ partira à $5 h$ n'est pas si précis que cela.

${ }^{2}$ Nous rappelons ici l'essentiel du propos de G. Kaltenböck, W. Mihatsch, S. Schneider exposé au 10th International Pragmatics Conference de Göteborg, juillet 2007.

${ }^{3}$ G. Lakoff (1972) a introduit le terme hedge : "words whose job it is to make things fuzzier".

${ }^{4}$ Voir l'article de Y. Homma (2005) sur l'approximation dénotée par par ici, par là, par là-bas.
} 
Quelques marqueurs de dérobade (et qui sont avant tout des marqueurs de discours) : je pense, je suppose, il semble que, il paraît, à mon avis, selon moi, personnellement...

Il n'existe pas à notre connaissance d'étude approfondie et étendue en français sur le "parler flou" / l'art de parler vague, et sur l'utilisation des marqueurs pour y arriver que ce soit en termes d'approximation ou de précision, mais des articles dispersés (H. Bat-Zeev Shyldkrot, J. Jayez, G. Kleiber, L. Melis, K. Ringenson, C. Vaguer, entre autres).

L'ensemble des articles du présent recueil permettront certainement de combler des manques sur l'identification et la caractérisation des marqueurs propres à dénoter l'approximation ou la précision. Notre contribution dresse, dans un premier temps, un état de l'approximation telle qu'abordée dans les grammaires et ouvrages de référence. Dans un second temps, l'adverbe facilement sera plus particulièrement examiné, d'abord à partir des dictionnaires, puis l'objectif sera de cibler davantage l'usage de facilement parmi les marqueurs d'approximation.

\section{TYPOLOGIE DE L'APPROXIMATION ${ }^{5}$}

Dans les grammaires et ouvrages de référence, la quantification en général et l'approximation en particulier ne sont évoquées ${ }^{6}$ que lorsque certaines catégories (adjectif indéfini, adverbe de quantité, déterminant...) lui font intrinsèquement référence, comme l'illustre cet extrait de R. L. Wagner \& J. Pinchon (1962 : 488) :

“sur" sert à construire les compléments qui évoquent d'une manière approximative le point ou le moment de la durée par rapport auquel on situe un événement.

Parmi les ouvrages consultés, seul P. Charaudeau traite de l'approximation en ellemême et pour elle-même ${ }^{7}$, du fait que sa grammaire s'efforce de "proposer des explications sémantiques de chacune des questions grammaticales traitées » (1992:5).

\subsection{Propriétés de l'approximation}

Pour P. Charaudeau, l'approximation se caractérise à l'aide de trois propriétés :

- la nature sémantique des mots auxquels s'applique l'approximation, à savoir l'appartenance sémantique aux êtres dénombrables - que ceux-ci soient matériels ou non.

- le mode de détermination de la quantité : elle est déterminée ${ }^{8}$ (ou précise) au même titre que la quantité exprimée par les noms de nombre du système arithmétique.

\footnotetext{
${ }^{5}$ Cette partie est une version modifiée de notre contribution au Colloque international de Strasbourg « La quantification et ses domaines ».

${ }^{6}$ Wagner \& Pinchon (1962), Grevisse (1993), Wilmet $\left(2003^{3}\right)$, entre autres.

${ }^{7}$ Voir le chapitre 5 intitulé La quantification et les quantificateurs dans la deuxième partie consacrée aux catégories de la langue (p. 237-277).

${ }^{8}$ Ce mode de détermination dans le domaine de la quantification connaît plusieurs variables :

- précise dans Il travaille cinq heures pas jour.

- imprécise dans Il travaille plusieurs heures pas jour.

- relative par rapport à une limite dans Elle boit trop de café.

- totalisante dans Elle a vendu tous ses livres.

- nulle dans Elle n'a vendu aucun livre.
} 
Sauf que la "quantité déterminée" peut être exprimée de façon "approximative"; elle devient alors une référence nombrée autour de laquelle se situe ("en plus" ou "en moins") la quantité évaluée. (Charaudeau, 1992 : 245)

- la variation du degré de quantité selon que celle-ci est forte ou faible.

La force ou la faiblesse de la quantité dépend du contexte et de la situation de discours, c'est-à-dire [...] des normes sociales qui déterminent le degré moyen à partir duquel doit être jugée l'importance de la quantité. (Charaudeau, 1992 : 239)

Ainsi trois indique un degré faible dans J'ai mangé trois cacahuètes au cocktail mais un degré fort dans J'ai acheté trois appartements sur Paris hier.

\subsection{Les marqueurs d'approximation}

Il existe en français moderne une grande diversité de marqueurs d'évaluation approximative ${ }^{9}$ (adverbes, prépositions, locutions prépositives...) qui sont étiquetés comme tels selon la valeur de leur référence nombrée. Ces dits marqueurs de quantification traduisent quatre valeurs pour la notion d'approximation :

a. elle peut correspondre à "plus ou moins" la référence donnée par le nombre, avec pour marqueurs spécifiques :

- la série des nombres en -aine : quarantaine, cinquantaine, etc.

- millier: Un millier de manifestants.

- dans les Numéral: Samson déjà a tué dans les deux mille adultes [Giraudoux]; Il a dans les quarante ans [Académie]; Les parents d'Ernestine mesurent l'un et l'autre dans les un mètre quatre-vingts [Vailland]; Cette voiture va chercher dans les vingt millions de centimes [Académie], etc.

- autres prépositions et adverbes : Environ cent francs; Aux environs de midi M. Joseph Caillaux descendait de son automobile; Autour de cent francs; La date se placerait aux alentours du 15 septembre; Il a obtenu quelque soixante pour cent des suffrages; Nous serons de retour vers dix heures; Sur les (coups de) huit heures, il sortit; A peu près; De l'ordre de; Quelque chose comme (ça), etc.

b. elle peut signaler les deux références entre lesquelles se situe la quantité. Les marqueurs sont :

- soit des numéraux coordonnés sans conjonction: Jusqu'à dix-onze heures [Willy et Colette]; On faisait travailler des enfants de six, sept ans [Queneau] ; J'ai deux-trois trucs à faire avant de partir, etc.

- soit des numéraux coordonnés avec conjonction (...ou..., entre...et...) : Combien de réponses avez-vous reçu? - Trente ou quarante mille. I-Entre trente et quarante mille.

- soit des nombres joints par à ou sa variante de...à... : Un filet d'eau de sept à huit pouces de profondeur [Chateaubriand] ; Je resterai quatre à cinq jours

\footnotetext{
${ }^{9}$ Inventaire extrait de C. Vaguer (à par.).
} 
chez le duc [Mérimée].

c. elle peut signaler un léger dépassement de la référence donnée par le nombre, avec pour marqueurs principaux :

- et quelques : On a mis trois jours et quelques pour arriver.

- bon(ne)(s): Il nous a fallu (trois bonnes heures / un bon quart d'heure) pour terminer ce travail.

- au moins : Il te faudra au moins deux jours pour te remettre.

- facilement : Il a facilement trente ans ${ }^{10}$.

- facile : Il a trente ans, facile.

d. elle situe la quantité en-deçà de la référence donnée par le nombre. Les marqueurs prototypiques sont :

- près de (degré fort), pas loin de: Il te faudra [près de / pas loin de] deux mois pour t'en remettre.

- à peine (degré faible) : Il te faudra à peine deux mois pour t'en remettre.

- tout au plus, au grand maximum: Il te faudra [tout au plus / au grand maximum] deux mois pour t'en remettre.

- presque :Il te faudra presque deux mois pour t'en remettre.

La grande diversité des marqueurs d'approximation empêche de les étudier tous dans une seule et même contribution. Ont été en particulier abordés les procédés de quantification marqués par les prépositions dans, vers et sur devant un numéral ${ }^{11}$. Ces marqueurs d'approximation se trouvent caractérisés par le fait qu'ils dénotent une "quantification non extensive", qu'ils entrent en distribution complémentaire tant du point de vue de leur comportement syntaxique (dans les numéral fait partie de constituants intraprédicatifs, les deux autres sont des GP ajouts de phrase) que sémantique (dans les numéral couvre un large éventail d'approximation - poids, mesure, âge, prix, heure - mais exclut l'approximation temporelle réservée à sur les coups de $X$ (approximation horaire) et vers (les) numéral pour les approximations temporelles de date, d'âge, de tranche horaire et d'heure).

L'adverbe facilement est également susceptible de marquer une quantification approximative avec un numéral : On te donnerait facilement dix-huit ans. Une étude sur corpus $^{12}$ permet d'avancer dans la caractérisation formelle (syntaxique et distributionnelle) et sémantique de cet emploi. Notre propos sera à l'interface entre la pragmatique, la sémantique et la syntaxe.

10 P. Charaudeau mentionne l'adverbe comme étant l'un des marqueurs qui «signalent un léger dépassement de la référence donnée par le nombre » (op. cit. :246). Il précise alors que l'emploi de facilement, dans ce contexte Il a facilement trente ans, relève du langage conversationnel.

11 Voir en particulier les contributions de C. Vaguer (2003, 2005, 2006, à par.) et de C. Vaguer, S.-U. Choi, M.-H. Jeong (2005).

${ }^{12}$ Il s'agit du corpus littéraire extrait de la base textuelle Frantext. Sur la période considérée (1840-2009), 5700 attestations de facilement ont pu être relevées parmi lesquelles une cinquantaine illustrent l'emploi approximatif de l'adverbe. 


\section{SI FACILEMENT M'ÉTAIT CONTÉ}

\subsection{Une approche lexicographique}

La consultation de dictionnaires contemporains permet d'avoir une idée des principaux sens retenus pour facilement et de conclure que cet adverbe reste dans l'ombre de l'adjectif facile dont il est dérivé. Trois significations principales sont attribuées à facilement (cf. la synthèse dans le Tableau 1 infra) :

\section{a. Avec facilité, sans effort, sans peine, aisément}

Pendant deux ans, je ne vins pas une seule fois à Paris, quoique les permissions $s$ 'accordassent bien facilement. (Renan)

On ne vient pas facilement à bout de cette peur irraisonnée. (Bernanos)

Nous devons tout faire pour cacher notre présence ici. Le moulin, établi sur le plateau de Grande-Vue, est trop facilement reconnaissable. (Verne)

Quand il [Théodose] était ému par un sentiment plus vif de colère, c'est alors qu'il se portait plus facilement à la clémence. (Bossuet)

Les princes oublient facilement la vertu et les services d'un homme éloigné. (Rollin)

\section{b. Pour peu de chose}

Il se vexe facilement.

Cette matière se casse facilement.

c. [Pour apprécier une quantité] Au moins

Il mettra facilement deux heures pour faire ce travail. (Lexis)

Il mettra facilement trois jours pour les retrouver. (Rey-Debove)

\begin{tabular}{|l|l|c|c|c|}
\hline & & $\begin{array}{c}\text { Sens1 } \\
\text { Sans peine }\end{array}$ & $\begin{array}{c}\text { Sens2 } \\
\text { Pour peu de } \\
\text { chose }\end{array}$ & $\begin{array}{c}\text { Sens3 } \\
\text { Au moins }\end{array}$ \\
\hline DUPRE - Trévise & 1972 & - & - & - \\
\hline GLLF - Larousse & 1973 & + & - & - \\
\hline LEXIS - Larousse & 1975 & + & - & + \\
\hline DFC - Larousse & 1980 & + & - & + \\
\hline TLF - CNRS & 1980 & + & - & + \\
\hline GDEL - Larousse & $1982-1985$ & + & + & - \\
\hline REY \& REY-DEBOVE - Robert & 1983 & + & + & - \\
\hline REY-DEBOVE - Robert & 1989 & + & + & + \\
\hline PECHOIN - Larousse & 1991 & + & - & - \\
\hline REY - Robert & 1993 & - & - & - \\
\hline Le Petit Larousse compact & 1997 & + & - & - \\
\hline
\end{tabular}

Tableau 1 - Significations de facilement retenues par les dictionnaires

L'approche lexicographique de facilement révèle que, si l'on arrive à dater l'apparition 
de facilement en $1475^{13}$, la diversité de ses emplois est peu reconnue. Le sens 1 reste, en effet, admis par l'ensemble de la communauté des lexicographes comme étant le sens commun, à privilégier. L'emploi approximatif de facilement (sens 3) n'est retenu, quant à lui, que par un nombre limité de dictionnaires, principalement édités chez Larousse : ce n'est qu'en 1975 que cet emploi fait son apparition dans le dictionnaire Lexis, et reste présent dans le Dictionnaire du français contemporain (1980), dans le Trésor de la langue française (1980) et dans le Dictionnaire méthodique du français actuel en 1989.

Comment expliquer que facilement voie apparaître, dans les années soixante, parmi ses emplois une valeur d'approximation? On laisse, bien évidemment, le soin de cette réponse aux spécialistes de l'histoire de la langue : une étude diachronique permettrait certainement de retracer l'évolution de ce terme et de voir quelles fonctions et quels sens étaient alors occupés par la forme à travers l'histoire. Seule une étude fine de sa grammaticalisation ${ }^{14}$ offrirait les réponses adéquates.

On peut cependant amorcer une première piste de réflexions en remontant aux origines latines du terme (Benoist \& Goelzer) du fait que des traces d'approximation sont repérables dans les signifiés de facio et facilis desquels est dérivé facilement. Le sens 2 de facio implique la signification de quantité et, plus précisément, celle de porter un jugement sur cette valeur, et il en va de même pour les sens 3 et 5 de facilis. Il n'est donc pas absurde de retrouver cette valeur parmi les emplois de facilement. Reste donc à savoir pourquoi facilement recouvre cet emploi qui ne lui était jadis pas réservé et à quelle autre forme il l'emprunte ${ }^{15}$.

\begin{tabular}{|l|l|}
\hline Facio & $\begin{array}{l}\text { 1. Etablir, poser, supposer, feindre } \\
\text { 2. Juger d'un certain prix, estimer, apprécier (facio parvi, minimi, pluris } \\
\text { = apprécier peu, très peu, davantage) } \\
\text { 3. Représenter } \\
\text { 4. Donner, appliquer } \\
\text { 5. ... }\end{array}$ \\
\hline Facilis & $\begin{array}{l}\text { 1. (Avec sens passif.) Qui peut se faire, qui se fait aisément, facile } \\
\text { 2. Commode à, bien disposé pour } \\
\text { 3. Qu'on se procure aisément, abondant, sans valeur } \\
\text { 4. Qui se fait heureusement, prospère } \\
\text { 5. (Avec sens actif.) Qui coûte peu, qui fait facilement (au prop. et au fig.), } \\
\text { adroit, souple, fécond } \\
\text { 6. Qui fait volontiers, porté à, enclin à ; apte à } \\
\text { 7. (Avec sens moyen.) Qui se lie facilement, traitable, sociable, complaisant, } \\
\text { doux; propice, peut se faire, qui se fait aisément, facile. }\end{array}$ \\
\hline
\end{tabular}

Tableau 2 - Significations de facio et facilis dans le dictionnaire de E. Benoist \& H. Goelzer

\footnotetext{
${ }^{13}$ Dans les Chroniques des chanoines de Neuchâtel : Doibt on facilement croire, cité par A. Hatzfeld \& A. Darmesteter.

14 Voir C. Marchello-Nizia (2006). Le terme de "grammaticalisation" désigne soit un phénomène de changement (phénomène de "grammaticalisation", processus de changement dynamique, et unidirectionnel, par lequel des mots lexicaux ou des constructions syntaxiques changent de statut et acquièrent un statut de forme grammaticale), soit l'étude théorique de ce changement (approche dite de la "grammaticalisation"), avec son cadre d'analyse, et une série de notions qui permettent de définir et d'interpréter, et donc de repérer et peut-être de prévoir, les différentes phases du changement en question.

${ }^{15}$ Un processus de collision homonymique entre facilement et une autre forme doit certainement se mettre en place mais il reste à l'identifier.
} 


\subsection{L'adverbe facilement comme marqueur d'approximation, propriétés distributionnelles}

Des contraintes permettent de caractériser l'emploi de facilement comme marqueur d'approximation. Parmi celles-ci, nous pouvons mentionner les restrictions sur les adverbes de degré, la coordination, la sélection des noms, la négation, l'interrogation qui distinguent ainsi les deux emplois de facilement (approximation vs manière).

\subsubsection{La non-compatibilité avec les adverbes de degré}

Facilement, en tant que marqueur d'approximation, ne peut être modifié par les adverbes de degré (2), ce qui n'est pas le cas de ses autres emplois (3). Facilement en (2) dénote donc, à lui seul, que le haut degré est atteint, mais surtout précise que facilement, dans cet emploi, retrouve la valeur qualitative de son sens de base.

(2) *Nous passons (plus / très / si / assez / trop) facilement deux heures pour mettre au point une phrase. [Fournier]

(3) Il ê̂t lavé (plus / très / si / assez / trop) facilement cinquante batées d'affilée qu'un seul pan de chemise. [Perret]

\subsubsection{La non-coordination}

Facilement, en tant que marqueur d'approximation, ne peut pas être coordonné avec un autre adverbe (4), ce qui n'est pas le cas de ses autres emplois (5).

(4) *L'expérience dura facilement et rapidement cinq à six secondes.

(5) Il eût lavé plus facilement et plus rapidement cinquante batées d'affilée qu'un seul pan de chemise.

\subsubsection{Les contraintes de sélection des noms et des verbes}

Facilement modifie essentiellement le déterminant numérique d'un GN. Le numéral est un nombre entier qui peut, ou non, être coordonné :

(6) a. Elle atteindrait ici facilement 1500 à 1700 kilos.

b. L'expérience dura facilement cinq à six secondes.

c. On y compte facilement 16 chromosomes clivés.

Les noms ${ }^{16}$ appartiennent au paradigme des noms dénombrables puisqu'ils acceptent une détermination numérale. Sont identifiées des unités de poids (kilos), de mesure du temps (seconde, heure, jour, semaine, an, année, mètre, centimètre), de monnaie (francs, drachmes), des êtres dénombrables (enfants, habitants) ou autres (chromosomes).

Une relation étroite existe donc entre les noms et les verbes. Le verbe, de par son sémantisme, sélectionne un $\mathrm{N}_{\text {approprié }}$ et, ce faisant, facilement est tributaire de ces contraintes de sélection :

\begin{tabular}{|l|l|l|}
\hline Unités & Noms & Verbes \\
\hline Poids & kilos & atteindre $_{[\text {kilos }]}$ \\
\hline
\end{tabular}

\footnotetext{
${ }^{16}$ L'analyse des $\mathrm{N}_{0}$ (nom en position sujet) n'apportent pas de caractérisation particulière : $57 \%$ des $\mathrm{N}_{0}$ sont de type "+hum" contre 43 \% de type "-hum".
} 


\begin{tabular}{|c|c|c|}
\hline & litre, verre & boire $_{[\text {litres, verres] }}$ \\
\hline Monnaie & $\begin{array}{l}\text { 'balles', drachmes, francs, } \\
\text { pistoles }\end{array}$ & $\begin{array}{l}\text { donner }_{[\text {balles] }}, \text { gagner }_{\text {[francs] }}, \\
\text { rapporter }_{\text {[drachmes] }} \text {, se vendre } \\
\text { trouver }_{[\text {francs] }} \text {, }\end{array}$ \\
\hline $\begin{array}{l}\text { Mesure de } \\
\text { temps }\end{array}$ & $\begin{array}{l}\text { seconde, minute, heure } \\
\text { jour, semaine, année, an }\end{array}$ & 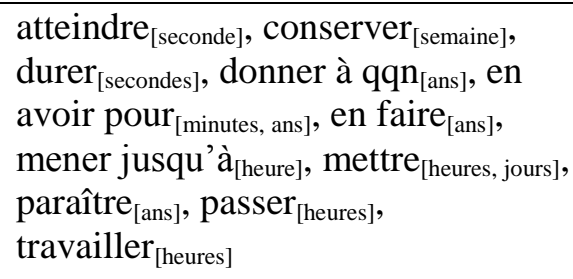 \\
\hline $\begin{array}{l}\text { Mesure de } \\
\text { longueur }\end{array}$ & centimètres, mètres, $\mathrm{km} / \mathrm{h}$ & $\begin{array}{l}\operatorname{arriver}_{[\mathrm{km} / \mathrm{h}]}, \text { assurer }_{[\mathrm{km} / \mathrm{h}]}, \\
\text { atteindre }_{[\text {mètre] }}, \text { dépasser }_{[\text {mètre] }}\end{array}$ \\
\hline $\begin{array}{l}\text { Etres } \\
\text { dénombrables }\end{array}$ & $\begin{array}{l}\text { élèves, enfants, habitants, } \\
\text { personnes, prisonniers }\end{array}$ & $\begin{array}{l}\text { avoir }_{[\text {personnes, élèves] }}, \text { contenir }_{[\text {habitants] }} \text {, } \\
\text { faucher }_{[\text {[enfants] }}, \text { mettre } \\
{[\text { [prisonniers] }}\end{array}$ \\
\hline $\begin{array}{l}\text { Autres } \\
\text { dénombrables }\end{array}$ & chromosomes, romans & avaler $_{\text {[romans] }}$, compter $_{\text {[chromosomes] }}$ \\
\hline
\end{tabular}

Tableau 3 - Noms \& Verbes dans les emplois approximatif de facilement d'après le corpus extrait de Frantext

\subsubsection{La négation, l'interrogation, la restriction "ne...que"}

On peut également reprendre les tests de P. Péroz (1992) dégageant la valeur "intensive" de bien dans des énoncés tels que Il est bien ridé, cet homme! (op. cit. : 58 sqq.) : la négation n'est pas bien tolérée (7), ni l'interrogation (8); c'est également le cas ici :

(7) ? On ne lui donnerait pas facilement dix-huit ans, dis donc!

(8) ? Est-ce qu'on lui donnerait facilement dix-huit ans?

à quoi on peut ajouter l'exclusion de la restriction ne...que :

(9) *On ne lui donnerait que facilement dix-huit ans.

L'ensemble de ces tests permettent de distinguer deux emplois de facilement (*Il ne mesure pas facilement 2 mètres vs. Il ne danse pas facilement) et de mettre en évidence que, avec facilement approximatif, on ne peut aller contre l'assertion.

\section{CARACTÉRISATION DE FACILEMENT}

(10) On te donnerait facilement dix-huit ans.

La difficulté est de séparer deux emplois possibles de facilement : (a) ou bien facilement porte sur donner (ajout au verbe) et est paraphrasable par Il est facile de te donner dixhuit ans; (b) ou bien facilement porte sur dix-huit ans (modificateur (ou spécifieur) du $\mathrm{GN}$ ) et est paraphrasable par Tu fais dix-huit ans bien sonnés.

Il semble que la différence sémantique est subtile, et qu'il faut à tout prix des tests clairs pour être sûr par la suite que l'on parle bien de l'emploi dit "d'approximation".

(i) Facilement est paraphrasable par de manière facile ou avec facilité, ou encore sans peine, ou à l'inverse difficilement, seulement dans l'emploi (a); l'emploi (b) l'éloigne donc de sa famille morphologique (i.e. des points communs sémantiques qu'il peut avoir avec ses autres membres) - cependant, l'adjectif 
facile adverbialisé serait ici possible avec la même interprétation ;

(ii) L'adverbe en général peut syntaxiquement se caractériser par sa place possible entre l'auxiliaire et le participe passé ou le semi-auxiliaire et l'infinitif: On t'aurait facilement donné dix-huit ans; dans cette position, il est impossible d'interpréter facilement au sens (b). Il en va de même pour facile, qui se comprend ici comme "il aurait été facile de te donner dix-huit ans" : On t'aurait facile donné dix-huit ans ;

(iii) Certains verbes ne se combinent guère avec facilement comme adverbe de manière ; ainsi, il n'y a pas d'ambiguïté dans Elle a facilement dix-huit ans, qui ne pourrait s'interpréter comme (a) et d'ailleurs ne tolère pas les paraphrases proposées ci-dessus, ni la position intra-verbale (*Elle a / aura / avait facilement eu dix-huit ans). On peut donc vérifier certaines manipulations en voyant ce que donne le test avec un verbe comme avoir. Un autre verbe témoin pourrait être paraître : Elle paraît facilement dix-huit ans $\left({ }^{*}\right.$ Elle paraît avec facilité dix-huit ans, *Elle avait facilement paru dix-huit ans, *Elle paraît sans peine dix-huit ans - cependant, ici, paraît relativement acceptable : ? Elle paraît difficilement dix-huit ans), ou encore Elle faisait facilement dix-huit ans (verbe faire dans son emploi attributif).

L'ensemble de ces propriétés permettent de différencier les deux principaux emplois de facilement et de révéler que dans le premier (sens 1 - manière) l'adverbe a une portée phrastique, ce qui n'est pas le cas pour le second (sens 2 - approximatif).

\subsection{Place et portée de l'adverbe}

Comme on sait, l'adverbe peut être soit “intégré à la proposition" (Molinier \& Levrier 2000), "intraprédicatif" (Guimier 1996), soit extraphrastique (adverbes souvent dits "d'énonciation"); du fait que facilement ne peut, au sens qui nous intéresse, apparaître en tête de phrase, il relève nécessairement de l'intraphrastique :

(11) a. On te donnerait facilement dix-huit ans.

b. *Facilement, on te donnerait dix-huit ans.

On s'attend alors à ce qu'il entre sous la portée de l'interrogation et de la négation, et soit clivable, or ce n'est pas ce que l'on observe (toujours au sens d'approximation quantitative) :

(12) a. *Est-ce qu'on lui donnerait facilement dix-huit ans ou au plus dix-huit ans?

b. *On ne te donnerait pas facilement dix-huit ans mais au plus dix-huit ans.

c. C'est facilement que l'on te donnerait dix-huit ans.

Si l'on suit C. Molinier (1990) et C. Molinier \& F. Levrier (2000), ces impossibilités confirment que facilement "approximatif" entre dans la sous-catégorie des adverbes quantitatifs, qui s'opposent précisément par là aux adverbes de manière ; dans le même ordre d'idées, facilement ne peut constituer une réponse à la question comment ? :

(13) *Comment lui donnerait-on dix-huit ans? - Facilement.

mais on peut l'associer à un adverbe quantitatif foyer d'une interrogative :

(14) Lui donnerait-on tout-à-fait / presque / à peine dix-huit ans? - On lui donnerait facilement dix-huit ans. 
En revanche, facilement n'est pas susceptible d'entrer dans la formation d'un déterminant, comme énormément (énormément de pain / *facilement de pain), mais il connaît une alternance avec l'adjectif adverbialisé (Il mange facilement / facile deux baguettes à chaque repas) alors que l'on n'aurait pas *Il mange énorme du pain.

Ce qui montre que facilement n'est pas un ajout au verbe, dans son emploi "approximation", c'est que l'adjectif facile ne peut modifier le prédicat nominalisé : si Il a pesé facilement les pommes de terre peut se paraphraser La pesée des pommes de terre fut facile, on n'a pas, face à Cette pomme de terre pèse facilement deux kilos, *La pesée / Le poids de cette pomme de terre fut facile, *La pesée / Le poids de deux kilos fut facile. En revanche, l'adverbe peut être associé lors du clivage au GN quantifié, ce qui montre leur solidarité syntaxique :

(15) a. Il mange énormément de pain / C'est énormément de pain qu'il mange.

b. On lui donnerait facilement dix-huit ans / C'est facilement dix-huit ans qu'on lui donnerait.

Cette propriété rapproche facilement des modaux (évidemment, apparemment...) qui, eux, relèvent de l'extraphrastique.

\subsection{La valeur de facilement}

Ces points communs peuvent être exploités sémantiquement si l'on admet qu'une propriété formelle a son corrélat du point de vue du sens : C. Molinier \& F. Levrier (Ibid.) en effet constatent que cette possibilité distributionnelle ne concerne parmi les modaux que ceux qui expriment le plus clairement la catégorie du nécessaire. Une hypothèse pour facilement au sens "approximation" est donc qu'il introduit la subjectivité que constitue l'évaluation sur le mode de l'aléthique: facilement présenterait les dix-huit ans comme un minimum nécessairement atteint.

Cette évidence que véhicule facilement dans cet emploi s'observe à la valeur qu'il peut prendre employé seul en réponse à une interrogation :

(16) Il doit bien peser 100 kilos ? - Oh, facilement! (sous entendu “c'est peu de le dire !", “ça, y a pas photo!", "aucun doute")

Le lien de l'adverbe, dans cet emploi, au sens de ses parents morphologiques : la facilité et le facile, s'opérerait alors du fait que "l'aisance", le "sans peine", l'évidence de la perception provient de son caractère nécessaire : on ne peut pas faire autrement que le voir, l'admettre comme vrai.

Autrement dit, facilement marque le renchérissement comme l'illustrent les énoncés sous (17). Cela rejoint donc l'analyse de H. Bat-Zeev Shyldkrot (2007) pour ce qui concerne l'évolution des champs sémantiques de certaines constructions :

Les items qui représentent la quantité moindre (peu, moins) deviennent des locutions exprimant une condition renversée pour peu que, à moins que. Les expressions qui traduisent une quantité supérieure, en revanche, en viennent à marquer le renchérissement (op. cit. : 9).

(17) a. J'en ai encore facilement pour trente ans à vivre.

b. J'en ai encore pour trente bonnes années à vivre.

c. J'en ai encore pour trente ans bien tassés à vivre.

Facilement, au-delà de marquer un degré de quantité - le numéral à lui seul illustre une quantité déterminée - traduit surtout une évaluation dans le jugement porté par le 
locuteur eu égard au contexte et à la situation d'énonciation ${ }^{17}$. Preuve en est, modalité (facilement) et quantification (trente à quarante minutes) peuvent se combiner sous (18) :

(18) Le henné, c'est facilement trente à quarante minutes avec les bras en l'air.

\section{CONCLUSION}

Cette première étude de facilement confirme la définition fournie par P. Charaudeau selon laquelle l'adverbe signale un léger dépassement de la référence donnée par le nombre. Facilement apporte son commentaire sur une valeur constante (le numéral), prise comme terme de référence. Il oriente l'interprétation de l'énoncé, suite à un premier constat (conscient ou inconscient) du locuteur : (i) je constate : "je sais que Paul a 18 ans", (ii) j'apporte un commentaire sur cette affirmation : "il a facilement 18 ans". Autrement dit, l'adverbe agit de l'intérieur et de l'extérieur par rapport au contenu phrastique ; «il contribue à construire ce contenu et simultanément, il le commente » (Guimier, 1996 : 13). Ainsi facilement se rapproche-t-il donc des modaux (évidemment, apparemment...) mais s'en distingue en introduisant la subjectivité que constitue l'évaluation sur le mode de l'aléthique (la valeur d'évidence, de nécessité, de vérité nécessaire): facilement présenterait les dix-huit ans comme un minimum nécessairement atteint.

Facilement, comme difficilement, apporte un commentaire sur un propos connu, qui ne peut être nié (i.e. il doit avoir une existence effective ${ }^{18}$ ). Il traduit donc l'attitude évaluative (le point de vue) du sujet parlant et, plus précisément, son renchérissement à l'égard de ce qui est dit (cf. Traugott \& Heine 1991, Shapira 2008). Il indique l'opinion affirmative qu'a le locuteur de la valeur de vérité de la proposition qu'il formule. Ainsi, au-delà d'être un marqueur de quantification - un quantifieur - facilement doit avant tout être identifié comme un marqueur de modalité aléthique (intensif-appréciatif) sur une quantification.

On peut se poser la question du rapport de cet emploi de facilement avec celui de l'adjectif adverbialisé facile :

(19) On met facile / facilement deux heures pour y arriver.

L'adjectif paraît avoir les mêmes propriétés distributionnelles et syntaxiques que l'adverbe, à ceci près que la postposition par rapport au GN paraît avec lui plus acceptable :

(20) Elle a pris dix kilos facile.

Elle a pris facile dix kilos.

Elle a pris facilement dix kilos.

?? Elle a pris dix kilos facilement.

Quel est le corrélat sémantique de cette propriété formelle ? Celle-ci montre en tous cas que l'on ne peut avoir dans l'adjectif adverbialisé une simple variante "populaire" de

\footnotetext{
${ }^{17}$ D'un point de vue sémantique donc, facilement est à identifier comme un adverbe endophrastique : «adverbe qui caractérise dans l'immanence même de l'énoncé une portion du monde référentiel auquel l'énoncé renvoie » (Guimier, $1996: 6$ et 96).

${ }^{18}$ Rappelons que facilement au sens "approximation" ne peut se combiner avec le morphème de négation, ni avec le futur ou le conditionnel: *Il n'a pas facilement dix-huit ans, ?? Il aurait facilement deux mètres, ?? Il aura facilement dix-huit ans.
} 
l'adverbe (Grevisse \& Goosse, 2008 : 1193).

Céline VAGUER

Université de Toulouse II-Le Mirail, Laboratoire CLLE-ERSS (CNRS UMR 5263)

\section{Références bibliographiques}

BAT-ZEEV SHYLDKROT H. (2007), "A peine et difficilement: concurrence et substitution", Colloque La Synonymie, Paris IV-Sorbonne.

CAFFI C. (1999), “On mitigation”, Journal of Pragmatics, 31, p. 881-909.

CAFFI C. (2001), La mitigazione. Un approccio pragmatico alla communicazione nei contesti terapeutici, Münster, LIT.

CAFFI C. (2007), Mitigation, Amsterdam, Elsevier.

CHARAUDEAU P. (1992), Grammaire du sens et de l'expression, Paris, Hachette Éducation.

FRASER B. (1975), "Hedged performatives", in P. Cole, J. L. Morgan, éds, Syntax and semantics, vol. 3, Speech acts, New York, Academic Press, p. 187-210.

GEZUNDHAJT H. (2000), Adverbes en '-ment' et opérations énonciatives, Bern, Peter Lang.

GREVISSE M. (1993), Le Bon usage. Grammaire française, Paris/Louvain-la-Neuve, Duculot (treizième édition refondue par A. Goosse, $1^{\text {re }}$ éd. 1936).

GREVISSE M. \& GOOSSE A. $\left(2008^{14}\right)$, Le Bon usage, Bruxelles, De Boeck/Duculot.

GROSS M. (1986), Grammaire transformationnelle du français. 3-Syntaxe de l'adverbe, Paris, ASSTRIL.

GUIMIER C. (1996), Les adverbes du français: le cas des adverbes en '-ment', Paris/Gap, Ophrys.

HOMMA Y. (2005), "La source de l'approximation dans par ici, par là, par là-bas", Linx, 53, p. 121-134.

JAYEZ J. (1987), "Sémantique et approximation : le cas de presque et à peine", Lingvisticae Investigationes, XI : 1, p. 157-196.

KALTENBÖCK G., MIHATSCH W., SCHNEIDER S. (2007), "The pragmatics of approximation : an introduction", Communication, 10th International Pragmatics Conference - IprA (8-13 juillet 2007), Göteborg.

KLEIBER G. (1987), "Quelques réflexions sur le vague dans les langues naturelles", Etudes de linguistique générale et de linguistique latine offertes en hommage à Guy Serbat, Paris, Bibliothèque de l'Information, p. 157-172.

LAKOFF G. (1972), "Hedges: A study in meaning criteria and the logic of fuzzy concepts", in P. M. Peranteau, J. N. Levi \& G. C. Phares, éds, Papers from the Eighth Regional Meeting of the Chicago Linguistic Society, Chicago, Chicago Linguistic Society, p. 183-228. [réed. en 1973 dans Journal of Philosophical Logic, 2, p. 458-508]. 
MARCHELLO-NIZIA C. (2006), Grammaticalisation et changement linguistique, Bruxelles, De Boeck \& Larcier, Coll. 'Champs linguistiques'.

MARTIN R. (1990), "Pour une approche vériconditionnelle de l'adverbe bien", Langue française, 88 , p. 80-89.

MELIS L. (2003), "Les quantificateurs approximatifs de type prépositionnel”, Verbum, $\mathrm{XXV}, 1$, p. 5-24.

MOLINIER C. (1990), "Une classification des adverbes en -ment", Langue française, 88 , p. $28-40$.

MOLINIER C., LEVRIER F. (2000), Grammaire des adverbes. Description des formes en '-ment', Genève, Droz.

NØLKE H., éd., (1990), Langue française $\mathrm{n}^{\circ} 88$ : Classification des adverbes, Paris, Larousse.

NØLKE H. (1990), "Les adverbiaux contextuels : problèmes de classification", Langue française, 88, p. 12-27.

PEROZ P. (1992), Systématique des valeurs de bien en français contemporain, Genève/Paris, Droz.

PRINCE E. F., FRADER J., BOSK C. (1982), "On Hedging in Physician-Physician Discourse", in R. J. Di Pietro, éd., Linguistics and the Professions, Norwood N.J., Ablex, p. 83-97.

RIEGEL M., PELLAT J.-C., RIOUL R. (1999), Grammaire méthodique du français, Paris, PUF ( $1^{\text {re }}$ éd. 1994).

RINGENSON K. (1940), "Il a dans les cinquante ans", Studier $i$ modern Sprakvetenskap, 14, p. 137-147.

SCHAPIRA C. (2008), "Focalisation discursive ou, plus précisément, exactitude", Communication à la Journée d'étude internationale 'Approximation et précision' $\left(1^{\text {er }}\right.$ juillet 2008), Tel-Aviv, Israël.

TRAUGOTT E.C., HEINE B. (1991), Approaches to grammaticalization, V, I-II, Amsterdam, Benjamins.

VAGUER C. (2003), “La préposition dans: vecteur d'approximation ?”, Revue de Sémantique et Pragmatique, 14, p. 135-155.

VAGUER C. (2005), "Dans les + numéral : un déterminant de quantification faible ?", Travaux de Linguistique, 50, p. 113-129.

VAGUER C. (2006), "Approche du sens des prépositions: le cas de vers", Modèles linguistiques, 54, XXVII-2, p. 37-50.

VAGUER C. (à par.), "Prépositions et quantification. Le cas de dans, vers et sur", Actes du Colloque international La quantification et ses domaines, Strasbourg (19-21 octobre 2006), France (à paraître aux Presses Universitaires de Caen dans la Coll. 'Syntaxe \& Sémantique').

VAGUER C., CHOI S.-U., JEONG M.-H. (2005), “L'approximation : un outil pour mettre en évidence des divergences dans l'emploi de la préposition dans en français et en coréen", BULAG, 30, p. 165-183. 
WAGNER R.-L., PINCHON J. (1962), Grammaire du français classique et moderne, Paris, Hachette.

WILMET M. $\left(2003^{3}\right)$, Grammaire critique du Français, Bruxelles, De Boeck \& Larcier Éds. Duculot (1 ${ }^{\mathrm{re}}$ éd. 1997).

\section{Dictionnaires}

DUBOIS C., GARNIER Y., CASALIS D. (1982-1985), Grand Dictionnaire Encyclopédique Larousse, Paris, Librairie Larousse, Dix volumes.

DUBOIS J. et coll. (1975), Lexis. Dictionnaire de la langue française, Paris, Librairie Larousse.

DUBOIS J. et coll. (1980), Dictionnaire du français contemporain, Paris, Librairie Larousse.

DUPRÉ P. (1972), Encyclopédie du bon français dans l'usage contemporain, Paris, Éditions de Trévise, Trois tomes.

EVENO B. (1997), Le Petit Larousse compact 1998, Paris, Larousse-Bordas.

GUILBERT L., LAGANE R., NIOBEY G. (1973), Grand Larousse de la Langue Française, Paris, Librairie Larousse, Six volumes.

PÉCHOIN D. (1991), Thésaurus Larousse. Des mots aux idées - Des idées aux mots, Paris, Larousse.

QUEMADA B. et coll. (1980), Trésor de la langue française. Dictionnaire de la langue du XIX et du XX siècle (1789-1960), Paris, Éditions du Centre national de la recherche scientifique, Seize volumes.

REY A., REY-DEBOVE J. (1983), Le Petit Robert 1. Dictionnaire alphabétique et analogique de la langue française, Paris, Le Robert.

REY A. (1993), Dictionnaire historique de la langue française, Paris, Dictionnaires Le Robert, Deux volumes.

REY-DEBOVE J. (1989), Dictionnaire méthodique du français actuel, Paris, Dictionnaires Le Robert.

\section{Autres dictionnaires consultés}

1778, Dictionnaire de l'Académie françoise, Nismes, chez P. Beaume.

AUGÉ C. (1922), Larousse universel en 2 volumes, Paris, Larousse.

AUGÉ C., Nouveau Larousse illustré, Paris, Larousse.

BEAUJEAN A. (1875), Dictionnaire de la langue française, abrégé du dictionnaire d'E. Littré, Paris.

BÉNARD M.-T. (1879), Dictionnaire classique universel, Paris, Librairie classique d'Eugène Belin.

BENOIST E., GOELZER H., Nouveau dictionnaire Latin-Français, Paris, Librairie Garnier Frères.

BESCHERELLE A. (1867), Dictionnaire national, Paris, Garnier Frères. 
BESCHERELlE A., BOURGUIGNON A. (1877), Dictionnaire usuel de la langue française, Paris, Garnier Frères.

BESCHERELLE A., PONS J.A. (1864), Nouveau dictionnaire de la langue française, Paris, Garnier Frères.

BOISTE P. (1829), Dictionnaire universel de la langue française, Paris, chez Verdière.

CATINEAU P. (1817), Nouveau dictionnaire de poche de la langue française, Paris, chez Lefèvre \& Tardieu-Denesle.

FLAMMARION C., Dictionnaire encyclopédique complet, Paris, Librairie des publications populaires.

HATZFELD A., DARMESTETER A., Dictionnaire générale de la langue française, Paris, Librairie Ch. Delagrave.

LACHATRE M., Nouveau dictionnaire universel, Paris, Édouard Blot.

LANDAIS N. (1850), Dictionnaire général et grammatical des dictionnaires français, Paris, Didier.

LARIVE M., FLEURY (1887), Dictionnaire français illustré des mots et des choses, Paris, Georges Chamerot.

LAROUSSE P. (1900), Dictionnaire complet illustré, Paris, Larousse.

LE TELLIER C. C. (1821), Nouveau dictionnaire portatif de la langue françoise, ou vocabulaire, Paris, chez Le Prieur \& Belin-Le Prieur.

LE TRÉVOUX (1721), Dictionnaire universel françois et latin, chez Delaulne.

LITTRÉ E. (1874), Dictionnaire de la langue française, Paris, Hachette.

NOËL M., CHAPSAL M. (1841), Nouveau dictionnaire de la langue française, Paris, Hachette.

\section{Sources}

[Frantext] http://www.frantext.fr

[Le Monde Diplomatique] Cédérom 1987-1997, Éditeur Cedrom-Sni. 